\title{
A Rare Collision Tumor: Tracheal Sarcomatoid Carcinoma Invading a Papillary Thyroid Carcinoma
}

\author{
Mofiyinfolu Sokoya ${ }^{*}$, Carissa M. Thomas ${ }^{1}$, Marcia Eustaquio ${ }^{1,2}$ \\ ${ }^{1}$ Department of Otolaryngology, School of Medicine, University of Colorado, Aurora, USA \\ ${ }^{2}$ Department of Otolaryngology-Head/Neck Surgery, Denver Health Medical Center, Denver, USA \\ Email: ^fiyin.sokoya@ucdenver.edu
}

How to cite this paper: Sokoya, M., Thomas, C.M. and Eustaquio, M. (2017) A Rare Collision Tumor: Tracheal Sarcomatoid Carcinoma Invading a Papillary Thyroid Carcinoma. Open Access Library Journal, 4: e4012.

https://doi.org/10.4236/oalib.1104012

Received: October 9, 2017

Accepted: December 19, 2017

Published: December 22, 2017

Copyright $\odot 2017$ by authors and Open Access Library Inc.

This work is licensed under the Creative Commons Attribution International License (CC BY 4.0).

http://creativecommons.org/licenses/by/4.0/

\begin{abstract}
A collision tumor involving a tracheal sarcomatoid carcinoma and a papillary thyroid carcinoma has never been reported in the literature. A 78-year-old female presented with a large neck mass and hoarseness. A fine needle aspiration demonstrated papillary thyroid carcinoma. Imaging revealed a right thyroid mass invading into the tracheal lumen with bulky ipsilateral lymphadenopathy. The patient underwent a total thyroidectomy, tracheal resection, and central and right neck dissections. Pathology demonstrated a tracheal sarcomatoid carcinoma invading the right thyroid, which had multifocal papillary thyroid carcinoma with central and lateral neck metastases. The patient subsequently received adjuvant chemoradiotherapy and radioactive iodine. We present a case of a tracheal sarcomatoid carcinoma colliding with a papillary thyroid cancer. Treatment modalities should address the most aggressive tumor, while being tailored to clinical picture, tumor histology, and patient preferences.
\end{abstract}

\section{Subject Areas \\ Oncology, Pathology}

\section{Keywords}

Collision Tumor, Spindle Cell Carcinoma, Sarcomatoid Carcinoma, Tracheal Malignancies, Thyroid Carcinoma

\section{Introduction}

Collision tumors are defined as two histologically distinct tumors co-localized in 
the same mass with collision of multiple tumor elements. These tumors can arise in the same organ, such as a combination of thyroid medullary and papillary carcinomas [1] [2], as a combination of tumors in adjacent organs, such as a collision between a bladder cancer and a sigmoid tumor [3], or as a combination of metastatic disease with a tumor in a primary organ [4]. Collision tumors of the thyroid gland have been previously described but are uncommon [1]. In this case presentation, we report a collision tumor comprised of a primary tracheal sarcomatoid carcinoma colliding with a papillary thyroid carcinoma (PTC).

Primary tracheal tumors are exceptionally rare and not widely reported in the literature. The incidence has been reported to be 0.2 per 100,000 people per year, accounting for less than $0.1 \%$ of cancer deaths [5]. Sarcomatoid carcinoma is a very rare variant of squamous cell carcinoma that accounts for less than $1 \%$ of all malignancies in the larynx [6]. The first case of tracheal sarcomatoid carcinoma was reported in 2009 [7]. This pathologic entity, also referred to as spindle cell carcinoma, pseudosarcoma, and carcinosarcoma, has been described in various body sites including the head and neck, upper and lower aerodigestive tract, skin, breast, and urogenital tract.

Given the rarity of tracheal tumors, especially the sarcomatoid variant, a collision tumor involving tracheal sarcomatoid carcinoma and PTC is extremely uncommon. Here, we present the first case of a patient with tracheal sarcomatoid carcinoma invading a PTC.

\section{Case Presentation}

A 78-year-old female presented to the emergency department with a large neck mass and hoarseness. A fine needle aspiration performed at an outside hospital demonstrated PTC. Flexible laryngoscopy was notable for paresis of the left true vocal fold and complete paralysis of the right true vocal fold. The tracheal lumen was not visible below the cords. The CT scan was notable for a large, heterogeneous, partially calcified mass measuring $4.8 \mathrm{~cm} \times 4.2 \mathrm{~cm} \times 5.2 \mathrm{~cm}$, arising from the right thyroid lobe with invasion of the right tracheal wall and protrusion into the tracheal lumen (Figure 1). There was an associated necrotic right zone III lymph node. A possible metastatic lesion was also found on the transverse process of the T1 vertebral body, confirmed on PET/CT. The patient was taken to the operating room for a total thyroidectomy with sleeve tracheal resectionand primary anastamosis and central and right neck dissections.

The final pathology demonstrateda sarcomatoid carcinoma of the trachea invading through the pretracheal fascia into the right thyroid gland (Figure 2). Immunohistochemical studies performed on the right thyroid lobe showed the sarcomatoid carcinoma to be diffusely and strongly positive for vimentin, and negative for pancytokeratin (AE1/AE3), CK5/6, CK19, CD30, ALK-1, CD15 and P63. There was strong positivity for EGFR in approximately $80 \%$ of cells, and very rare focal positivity for p16 in around $1 \%$ of cells. The thyroid gland was noted to have multifocal well differentiated PTC. No evidence was 

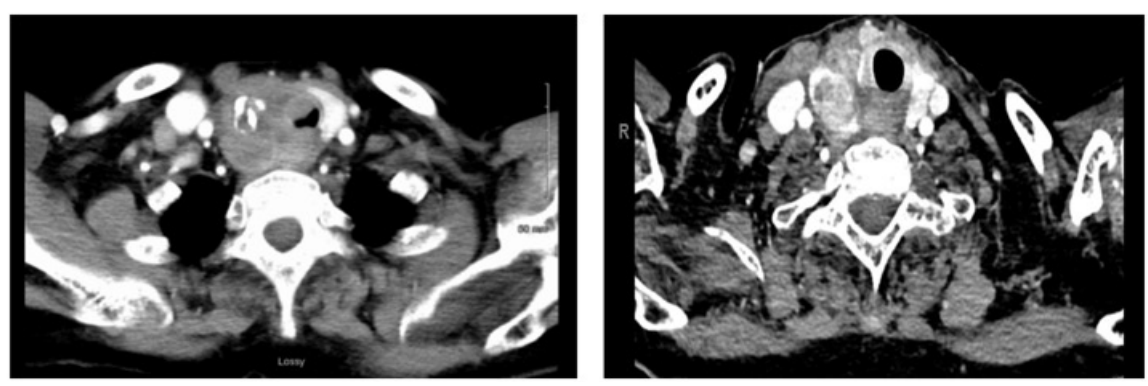

Figure 1. Two slices of a non-contrasted CT scan of the neck showing a large, heterogeneous, partially calcified mass involving the right thyroid lobe and the right tracheal wall, with deviation of the trachea and extension into the lumen.

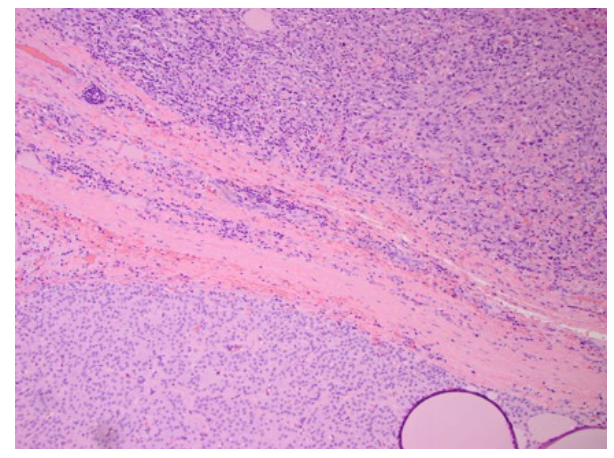

Figure 2. Low power view of the edge of the superior right thyroid lobe, showing benign thyroid tissue on bottom, fibrous capsule in middle, and infiltrating sarcomatoid carcinoma that was investing the thyroid on top. Magnification: $10 \times$. H\&E staining.

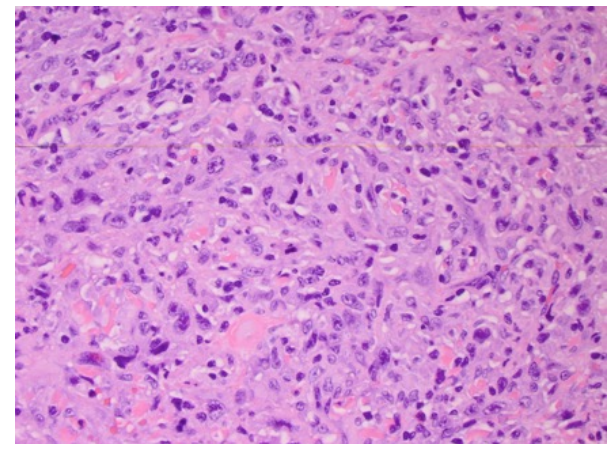

Figure 3. High power view of the area of infiltrating sarcomatoid carcinoma from Figure 2. Magnification: 40×. H\&E staining.

found for anaplastic thyroid carcinoma. One central neck node and one level II lymph node were positive for PTC. Additionally, one right level IV lymph node was positive for sarcomatoid carcinoma. There was no extracapsular extension (ECE). The tracheal sarcomatoid carcinoma was staged as T4aN1M0 and the PTC was staged as T1bN1bM0. The patient subsequently received adjuvant chemotherapy with carbotaxol and radiation to a total dose of $6600 \mathrm{~Gy}$ as well as radioactive iodine. Her right true vocal fold remained paretic, and she underwent a temporary injection medialization laryngoplasty of the right vocal fold, 
and eventually a type 1 thyroplasty. She has a satisfactory voice outcome.

\section{Discussion}

Collision tumors are defined as two histologically distinct neoplasms occupying the same mass. Multiple hypotheses have been proposed to explain the pathogenesis of this rare entity. One theory is the "chance theory", which proposes that two tumors come to occupy the same mass purely by chance. The mechanisms of tumorigenesis of the two tumors occur independently, and the geographical proximity of the neoplasms is coincidental. Another theory is the "neoplastic coercion theory" in which an initial tumor can promote the development of another tumor by means of creating a permissive local environment [8]. Lastly is the concept of a pluripotent progenitor, in which a common precursor has differentiated into two distinct histologic entities both of which become malignant [9]. This report represents a case of a collision tumor in the thyroid with cell types of distinct embryologic origins suggesting the tumor arose either by the "chance theory" or the "neoplastic coercion theory".

Primary malignant tracheal tumors are extremely rare and comprise a mere $0.2 \%$ of all malignant tumors of the respiratory tract [10]. Sarcomatoid carcinoma of the trachea is even more rare, with the first case being reported in 2009 in Turkey [7]. This case report is the second reported case of a tracheal spindle cell carcinoma. The pathology, course, and prognosis of spindle cell carcinoma is a subject of much controversy. There are sarcomatoid elements and squamous elements in the spindle cell carcinoma, and there are multiple theories addressing the pathogenesis of this tumor. These include the "collision theory", the "combination theory", and the "conversion theory" [11]. The "collision theory" of spindle cell carcinoma states that the sarcomatoid tumor elements arise independently from the squamous elements. The "combination theory" purports that both elements arose from a pluripotent stem cell that has the ability to differentiate along both lines. The "conversion theory" is based on the hypothesis that the sarcomatoid element derives from the squamous element during the tumor's growth. Spindle cell carcinomas are thought to occur primarily in male smokers in their $7^{\text {th }}-8^{\text {th }}$ decades of life. In a review of laryngeal and hypopharyngeal spindle cell carcinomas conducted by Lambert et al., age at diagnosis ranged from 42 to 87 years, with a mean of 64 years and male to female ratio of 10:1 [12]. However, in another case report series, females predominated 1.5 to 1 with a mean age of 61 years [13].

PTC is the most common thyroid malignancy, representing about $80 \%$ of all thyroid malignancies, and its treatment is well established. The overall survival rate is over $90 \%$ [14]. The recommended first line treatment is surgical excision. Radioactive iodine may then be used depending on the size, location, and characteristics of the tumor. Surgical resection of sarcomatoid carcinomas is more often recommended over radiation therapy. Some studies have shown that radiation therapy is associated with frequent recurrences and is not effective as the 
sole modality of treatment [15] [16]. However, there are other reports of complete response to primary chemoradiotherapy [13].

In our case, the patient underwent complete surgical excision followed by adjuvant chemoradiotherapy (total dose of $6600 \mathrm{~Gy}$ ) with carbotaxol followed by radioactive iodine. In collision tumors, treatment should be dictated by the most aggressive tumor histology as was the case in our patient.

\section{Conclusion}

Collision tumors are uncommon; and those involving rare tumors are exceptionally rare. We describe a tracheal sarcomatoid carcinoma in combination with a PTC that was treated with surgical excision followed by chemoradiotherapy targeting the sarcomatoid carcinoma and eventually radioactive iodine for the PTC. Treatment planning should be done in a multidisciplinary fashion and should address the most aggressive tumor first, while still accounting for clinical picture, tumor histology, and patient preferences.

\section{Disclosures}

MS has no conflicts of interests to disclose, CMT has no conflicts of interests to disclose, ME has no conflicts of interests to disclose.

\section{References}

[1] Brandwein-Gensler, M., Urken, M. and Wang, B. (2004) Collision Tumor of the Thyroid: A Case Report of Metastatic Liposarcoma plus Papillary Thyroid Carcinoma. Head Neck, 26, 637-641. https://doi.org/10.1002/hed.20024

[2] Pastolero, G.C., Coire, C.I. and Asa, S.L. (1996) Concurrent Medullary and Papillary Carcinomas of Thyroid with Lymph Node Metastases. A Collision Phenomenon. The American Journal of Surgical Pathology, 20, 245-250. https://doi.org/10.1097/00000478-199602000-00014

[3] Oda, Y., Hamami, G., Umezu, K., et al. (1984) Vesicocolic Fistula Formed by "Collision" Tumor between Transitional Cell Carcinoma of Urinary Bladder and a. Hinyokika Kiyo, 30, 55-58.

[4] Fornelli, A., Bacci, A., Collina, G. and Eusebi, V. (1995) Breast Carcinoma Metastatic to Meningioma: Review of the Literature and Description of 2 New Cases. $\mathrm{Pa}$ thologica, 87, 506-512.

[5] Li, W., Ellerbroek, N.A. and Libshitz, H.I. (1990) Primary Malignant Tumors of the Trachea. A Radiologic and Clinical Study. Cancer, 66, 894-899. https://doi.org/10.1002/1097-0142(19900901)66:5<894::AID-CNCR2820660514>3.0 .CO;2-W

[6] Hellquist, H. and Olofsson, J. (1989 Spindle Cell Carcinoma of the Larynx. APMIS, 97, 1103-1113. https://doi.org/10.1111/j.1699-0463.1989.tb00524.x

[7] Aksu, K., Aktaş, Z., Aksu, F., et al. (2009) Spindle Cell Sarcomatoid Carcinoma: First Case Reported in the Trachea. Tuberk Toraks, 57, 337-341

[8] Ryan, N., Walkden, G., Lazic, D. and Tierney, P. (2015) Collision Tumors of the Thyroid: A Case Report and Review of the Literature. Head Neck, 37, E125-E129. https://doi.org/10.1002/hed.23936 
[9] Lax, S.F., Beham, A., Kronberger-Schönecker, D., Langsteger, W. and Denk, H. (1994) Coexistence of Papillary and Medullary Carcinoma of the Thyroid Gland-Mixed or Collision Tumour? Clinicopathological Analysis of Three Cases. Virchows Archiv, 424, 441-447. https://doi.org/10.1007/BF00190568

[10] Honings, J., van Dijck, J.A., Verhagen, A.F., van der Heijden, H.F. and Marres, H.A. (2007) Incidence and Treatment of Tracheal Cancer: A Nationwide Study in the Netherlands. Annals of Surgical Oncology Website, 14, 968-976.

https://doi.org/10.1245/s10434-006-9229-Z

[11] McCluggage, W.G. (2002) Malignant Biphasic Uterine Tumours: Carcinosarcomas or Metaplastic Carcinomas? Journal of Clinical Pathology, 55, 321-325. https://doi.org/10.1136/jcp.55.5.321

[12] Lambert, P.R., Ward, P.H. and Berci, G. (1980) Pseudosarcoma of the Larynx: A Comprehensive Analysis. Archives of Otolaryngology, 106, 700-708.

https://doi.org/10.1001/archotol.1980.00790350042012

[13] Colozza, M., Grignani, F., Crino, L., Tonato, M. and Davis, S. (1988) Metastatic Spindle Cell Carcinoma: A Complete Response Induced by Cisplatin and 5-Fluorouracil. Anticancer Research, 8, 457-458.

[14] Caron, N.R. and Clark, O.H. (2006) Papillary Thyroid Cancer. Current Treatment Options in Oncology, 7, 309-319. https://doi.org/10.1007/s11864-006-0040-7

[15] Leventon, G.S. and Evans, H.L. (1981) Sarcomatoid Squamous Cell Carcinoma of the Mucous Membranes of the Head and Neck: A Clinicopathologic Study of 20 Cases. Cancer, 48, 994-1003.

https://doi.org/10.1002/1097-0142(19810815)48:4<994::AID-CNCR2820480424>3.0 .CO;2-M

[16] Benninger, M.S., Kraus, D., Sebek, B., Tucker, H.M. and Lavertu, P. (1992) Head and Neck Spindle Cell Carcinoma: An Evaluation of Current Management. Cleveland Clinic Journal of Medicine, 59, 479-482. https://doi.org/10.3949/ccjm.59.5.479 